\title{
Response to nivolumab in metastatic collecting duct carcinoma expressing PD-L1: A case report
}

\author{
KOSUKE MIZUTANI $^{1}$, KENGO HORIE ${ }^{1}$, SHINGO NAGAI ${ }^{1}$, TOMOHIRO TSUCHIYA ${ }^{1}$, \\ CHIEMI SAIGO $^{2}$, KAZUHIRO KOBAYASHI ${ }^{2}$, TATSUHIKO MIYAZAKI ${ }^{2}$ and TAKASHI DEGUCHI ${ }^{1}$ \\ ${ }^{1}$ Department of Urology, Gifu University Graduate School of Medicine; \\ ${ }^{2}$ Pathology Division, Gifu University Hospital, Gifu, Gifu 501-1194 Japan
}

Received May 30, 2017; Accepted August 16, 2017

DOI: $10.3892 / \mathrm{mco} .2017 .1449$

\begin{abstract}
The authors present a case report of collecting duct carcinoma (CDC) that responded to nivolumab, a programmed death 1 (PD-1) immune-checkpoint-inhibitor antibody, following the failure of systemic treatment with chemotherapy and targeted therapy. The patient underwent right radical nephrectomy and segmentectomy of the lung following chemotherapy. Fifteen months following the first surgery, segmentectomy and subsequent second-line chemotherapy were performed for recurrence in the lung. Targeted therapy with temsirolimus for recurrence of the lung and lymph node metastases was ultimately used for 30 months. However, the temsirolimus treatment failed to suppress the growth of metastatic lesions. Nivolumab resulted in complete response of the lung metastasis, and it stabilized the lymph node metastasis. PD-L1 was highly expressed in both primary tumor and the metastatic regions. Therapy with nivolumab is ongoing. These findings suggest that treatment with nivolumab may be considered for metastatic and treatment-failure CDC.
\end{abstract}

\section{Introduction}

Collecting duct carcinoma (CDC) is a rare variant subtype of renal cell carcinoma (RCC) known to originate in the distal collecting duct and to have an extremely poor prognosis, which is reported to be due to limited success with chemotherapy and targeted therapy $(1,2)$.

Correspondence to: Dr Kosuke Mizutani, Department of Urology, Gifu University Graduate School of Medicine, 1-1 Yanagido, Gifu, Gifu 501-1194, Japan

E-mail: mizutech@gifu-u.ac.jp

Abbreviations: CDC, collecting duct carcinoma; PET/CT, positron emission tomography/computed tomography; PD-1, programmed death 1 ; RCC, renal cell carcinoma

Key words: nivolumab, PD-1, PD-L1, collecting duct carcinoma, non-clear renal cell carcinoma
Programmed death 1 (PD-1) is a receptor expressed on activated T cells and binds to its ligands PD-L1 and PD-L2, which are present on antigen-presenting cells and tumor cells. PD-1 inhibitor blocks the interaction of PD-1 and its ligands and eventually restores antitumor immunity. Nivolumab improves overall survival in several cancers including $\operatorname{RCC}(3,4)$. It was reported that the expression of PD-L1 correlates with the effect of nivolumab in metastatic melanoma and some types of lung cancer, but the correlation is unclear in clear cell RCC (3-5). Although nivolumab improved overall survival of patients with antiangiogenic treatment-relapsed RCC with a clear-cell component compared with everolimus, the usefulness of nivolumab for CDC is unclear.

In the present study, we report a case showing a favorable response to nivolumab in metastatic $\mathrm{CDC}$. We also show the expression of PD-L1 in surgical specimens.

\section{Case report}

A 64-year-old man with a past history of papillary adenocarcinoma of thyroid cancer presented with bilateral lung tumors that were detected incidentally on positron emission tomography/computed tomography (PET/CT) for systemic evaluation of post-treatment thyroid cancer. PET/CT also revealed a right renal mass. A dynamic contrast-enhanced CT scan suggested that the renal tumor mass may be a metastatic renal tumor or non-clear RCC (Fig. 1). The patient underwent a core biopsy of the renal mass, and histological examination of the specimen was interpreted as carcinoma of renal origin. The patient had also undergone laparoscopic right radical nephrectomy and video-assisted thoracoscopic segmentectomy of the right lung at the same time. The left lung tumor was not resected because it was too small to conclude that it was metastasis, and it was followed with careful watching. Pathology diagnosed the renal tumor and resected lung tumor to be CDC and metastasis of CDC, respectively. Subsequently, four courses of chemotherapy with a combination of gemcitabine and cisplatin or carboplatin were administered as adjuvant therapy (Fig. 2).

Fifteen months after the first surgery, a follow-up CT scan showed that the left lung mass had increased in size. Video-assisted thoracoscopic segmentectomy of the left lung was performed, and this tumor was also diagnosed 


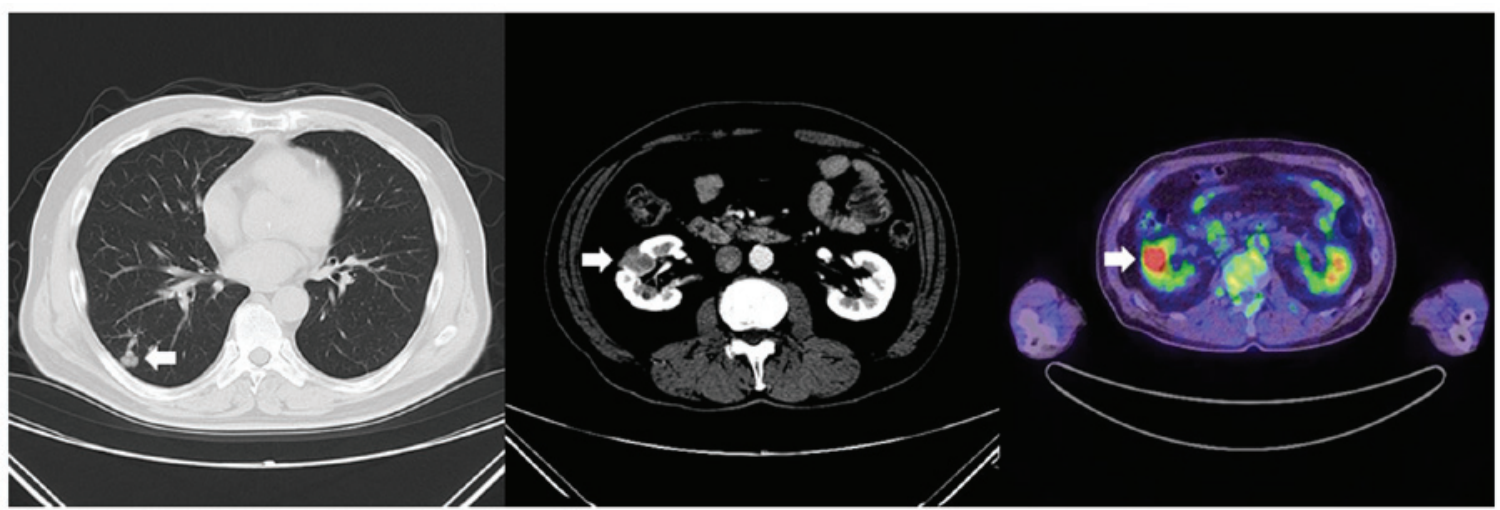

Figure 1. Computed tomography and positron emission tomography/computed tomography images showing a right renal mass and lung tumors (arrows).

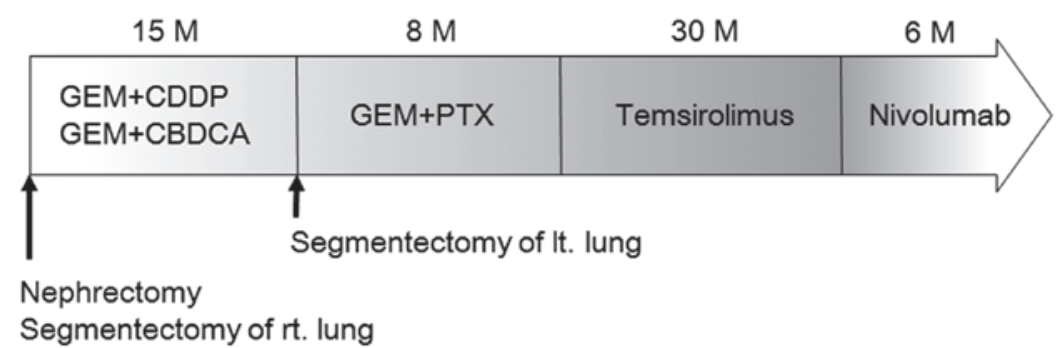

Figure 2. Schematic diagram of sequential treatment. GEM, gemcitabine; CDDP, cisplatin; CBDCA, carboplatin; PTX, paclitaxel; M, months.

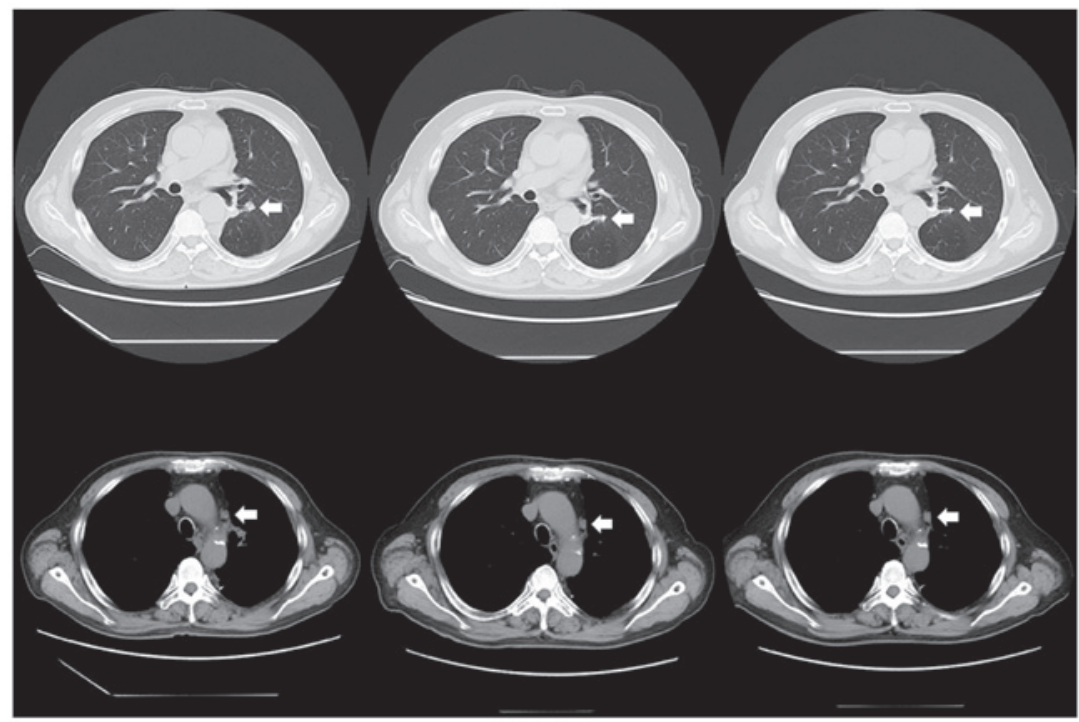

Figure 3. Therapeutic response. Chest computed tomography images show complete response of the left lung lesion and stable disease of the lymph node metastasis after nivolumab treatment (arrows). Left, before treatment; middle, 3 months after treatment; right, 6 months after treatment.

as metastasis of CDC. Pathology also revealed a small metastasis in the specimen of left lung; therefore, second-line chemotherapy with a combination of gemcitabine and paclitaxel was administered to the patient. A follow-up CT scan showed incidences of mediastinal lymph node and lung metastasis. The patient was treated with temsirolimus, and the metastasis remained at the same size for 30 months. However, a follow-up CT scan indicated increased size of the lung and lymph node metastases. The patient was then deemed a candidate for immune checkpoint inhibitor therapy with nivolumab. Six months after the treatment with nivolumab, a CT scan showed complete response of the lung metastasis and stable disease of the lymph node metastasis (Fig. 3). The therapy with nivolumab is ongoing with no adverse events.

To the best of our knowledge, there are no reported studies of immune checkpoint inhibitor therapy and PD-L1 expression in CDC. We elucidated the expression of PD-L1 by immunohistochemistry using anti-PD-L1 (clone 28-8) antibody. As 


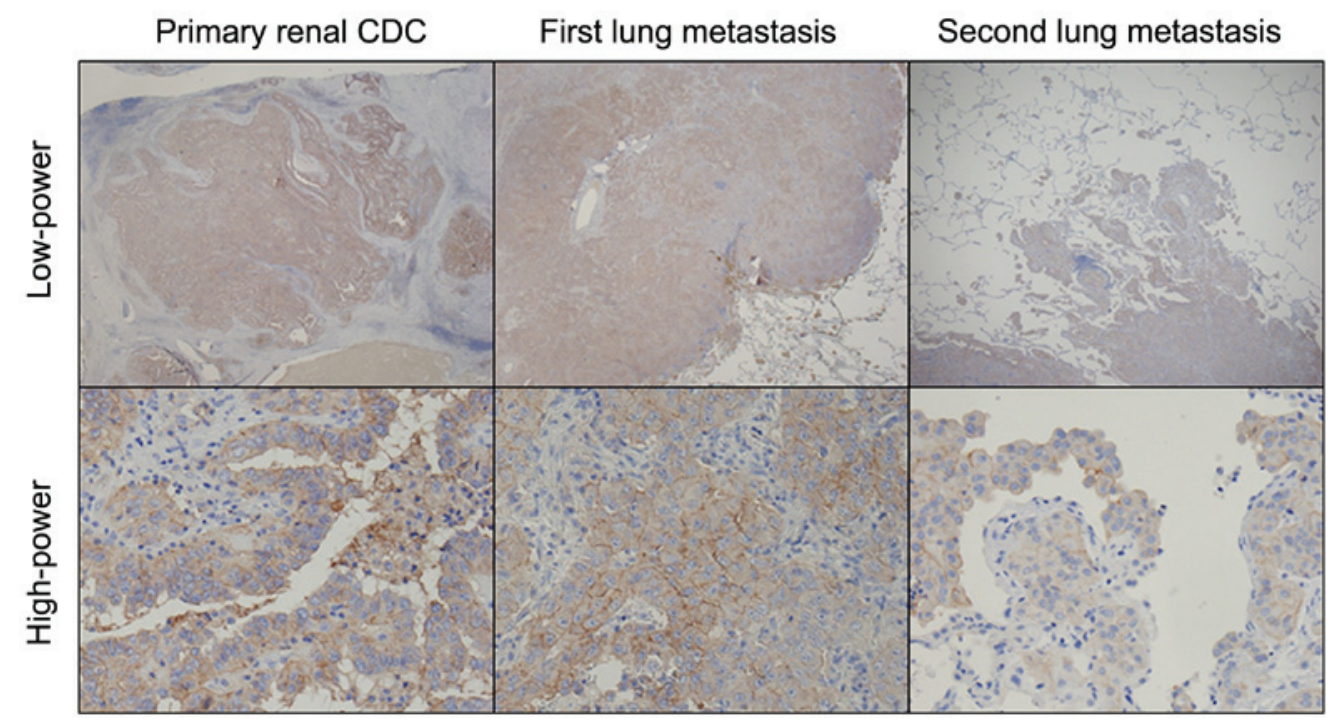

Figure 4. Low- and high-power microscopic views of the expressions of PD-L1 in surgical specimens as demonstrated by immunohistochemistry. Left column: Primary renal collecting duct carcinoma (CDC), middle column, first lung metastasis; right column, second lung metastasis; low-power, original magnification $\mathrm{x} 20$; high power, original magnification $\mathrm{x} 200$.

shown in Fig. 4, PD-L1 was highly expressed in all the surgical specimens (primary renal collecting duct carcinoma, first lung metastasis and second lung metastasis).

Written informed consent was obtained from the patient. The study was approved by the Ethics Committee of Gifu University, Graduate School of Medicine (approval no. 29-303).

\section{Discussion}

Rimar et al reported that nivolumab induced a clinical response to metastatic CDC without evaluating PD-1 or PD-L1 expression (6). To the best of our knowledge, the present report is the first to show that nivolumab induces a clinical response in metastatic CDC with evaluation of PD-L1 expression in surgical specimens.

Although it is not clear whether PD-L1 expression in surgical specimens is a predictive biomarker, some researchers have interpreted a high level of PD-L1 expression as possibly correlating with the effect of anti-PD-1/PD-L1 antibodies in several types of cancer (7). In a study on advanced RCC treated with nivolumab or everolimus, the median overall survival was 21.8 months in patients with $>1 \%$ PD-L1 expression and 27.4 months in patients with $<1 \%$ PD-L1 expression in the nivolumab group (4). Although the expression level of PD-L1 and its role in cancer prognosis remain to be elucidated, previous findings have shown that PD-L1 expression may vary in cancer types, and a high expression of PD-L1 is associated with poor clinical outcomes including that from clear cell RCC (8-10). On the basis of the mechanism of immune checkpoint inhibitor therapy, nivolumab to contribute to the improvement of clinical outcome in cancer that is exacerbated by the increased expression of PD-L1. The findings in the present case indicate to us that a high expression of PD-L1 in lung metastasis of $\mathrm{CDC}$ to contribute to a favorable clinical response to nivolumab. Further studies may demonstrate the effect of nivolumab on CDC and the correlation of PD-L1 expression with its benefit for $\mathrm{CDC}$ are anticipated.

\section{References}

1. Chao D, Zisman A, Pantuck AJ, Gitlitz BJ, Freedland SJ, Said JW, Figlin RA and Belldegrun AS: Collecting duct renal cell carcinoma: Clinical study of a rare tumor. J Urol 167: 71-74, 2002.

2. Yin M, Wang W, Rosenberg J, Kaag M, Joshi M, Holder S, Tuanquin L and Drabick JJ: Targeted therapy in collecting duct carcinoma of the kidney: A case report and literature review. Clin Genitourin Cancer 14: e203-e206, 2016.

3. Borghaei H, Paz-Ares L, Horn L, Spigel DR, Steins M, Ready NE, Chow LQ, Vokes EE, Felip E, Holgado E, et al: Nivolumab versus docetaxel in advanced nonsquamous non-small-cell lung cancer. N Engl J Med 373: 1627-1639, 2015.

4. Motzer RJ, Escudier B, McDermott DF, George S, Hammers HJ, Srinivas S, Tykodi SS, Sosman JA, Procopio G, Plimack ER, et al: Nivolumab versus Everolimus in advanced renal-cell carcinoma. N Engl J Med 373: 1803-1813, 2015.

5. Robert C, Long GV, Brady B, Dutriaux C, Maio M, Mortier L, Hassel JC, Rutkowski P, McNeil C, Kalinka-Warzocha E, et al: Nivolumab in previously untreated melanoma without BRAF mutation. N Engl J Med 372: 320-330, 2015.

6. Rimar KJ, Meeks JJ and Kuzel TM: Anti-programmed death receptor 1 blockade induces clinical response in a patient with metastatic collecting duct carcinoma. Clin Genitourin Cancer 14: e431-e434, 2016.

7. Zhang T, Xie J, Arai S, Wang L, Shi X, Shi N, Ma F, Chen S, Huang L, Yang L, et al: The efficacy and safety of anti-PD-1/PD-L1 antibodies for treatment of advanced or refractory cancers: A meta-analysis. Oncotarget 7: 73068-73079, 2016.

8. Erlmeier F, Weichert W, Schrader AJ, Autenrieth M, Hartmann A, Steffens S and Ivanyi P: Prognostic impact of PD-1 and its ligands in renal cell carcinoma. Med Oncol 34: 99, 2017.

9. Wang X, Teng F, Kong L and Yu J: PD-L1 expression in human cancers and its association with clinical outcomes. Onco Targets Ther 9: 5023-5039, 2016.

10. Xu F, Xu L, Wang Q, An G, Feng G and Liu F: Clinicopathological and prognostic value of programmed death ligand-1 (PD-L1) in renal cell carcinoma: A meta-analysis. Int J Clin Exp Med 8: 14595-14603, 2015. 